\title{
Molluscan Fossils and Stratigraphic Descriptions from the Upper Cretaceous Mancos Shale, West-Central Colorado
}

Open-File Report 2006-1326 


\section{Molluscan Fossils and Stratigraphic Descriptions from the Upper Cretaceous Mancos Shale, West-Central Colorado}

By E.A. Merewether, D.A. Sawyer, and W.A. Cobban

Open-File Report 2006-1326 


\section{U.S. Department of the Interior DIRK KEMPTHORNE, Secretary}

\section{U.S. Geological Survey \\ Mark D. Myers, Director}

\section{U.S. Geological Survey, Reston, Virginia: 2006}

For product and ordering information:

World Wide Web: http://www.usgs.gov/pubprod

Telephone: 1-888-ASK-USGS

For more information on the USGS — the Federal source for science about the Earth, its natural and living resources, natural hazards, and the environment:

World Wide Web: http://www.usgs.gov

Telephone: 1-888-ASK-USGS

Any use of trade, product, or firm names is for descriptive purposes only and does not imply endorsement by the U.S. Government.

Although this report is in the public domain, permission must be secured from the individual copyright owners to reproduce any copyrighted materials contained within this report.

Suggested citation:

Merewether, E.A., Sawyer, D.A., and Cobban, W.A., 2006, Molluscan fossils and stratigraphic descriptions from the Upper Cretaceous Mancos Shale, west-central Colorado: U.S. Geological Survey Open-file Report 2006-1326, 17 p, 


\section{Contents}

Introduction

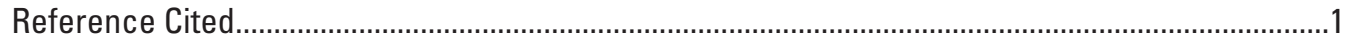

Outcrop Descriptions

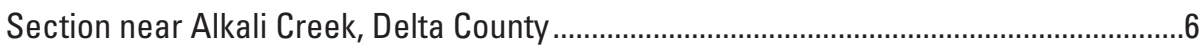

Section near Village of Hotchkiss, Delta County ..............................................................8

Section near Village of Mack, Mesa County...................................................................

Section near Olathe, Montrose County.............................................................................11

Section in Peach Valley, Delta County ...........................................................................12

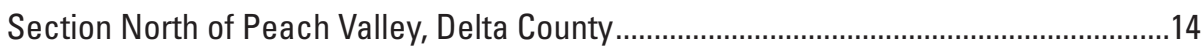

Section near Red Rock Canyon, Montrose County.........................................................15

Section near Uncompahgre, Montrose County ...........................................................16

\section{Figure}

1. Outcrop sections and fossil locatities in Upper Cretaceous strata in

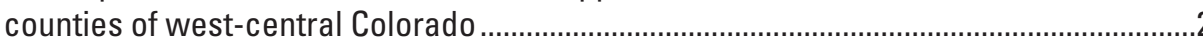

\section{Tables}

1. Members of Upper Cretaceous age in the lowest part of the Mancos Shale of west-central Colorado

2. Names and locations of selected outcrops of Upper Cretaceous strata and names of associated molluscan fossils in Delta, Mesa, and Montrose Counties, west-central Colorado $\ldots 4$

3. Molluscan fossil localities in Upper Cretaceous strata in Delta, Garfield, Mesa, and Montrose Counties, west-central Colorado..... 


\title{
Molluscan Fossils and Stratigraphic Descriptions from the Upper Cretaceous Mancos Shale, West-Central Colorado
}

\author{
By E.A. Merewether, D.A. Sawyer, and W.A. Cobban
}

\section{Introduction}

This report is based on lithostratigraphic and biostratigraphic data derived from investigations of Upper Cretaceous strata in Delta, Garfield, Mesa, and Montrose Counties in west-central Colorado (fig. 1). The data were obtained by personnel of the U.S. Geological Survey in the years 1955 through 2004, during studies of the Dakota Sandstone and the overlying Mancos Shale. Described herein are exposed strata of marine origin in the uppermost Dakota and the lower part of the Mancos (Cenomanian, Turonian, and Coniacian Stages) and related and associated collections of molluscan fossils (tables 1, 2, and 3).

The stratigraphic nomenclature used in this report for the lower part of the Mancos follows that of Molenaar and others (2002), who divided the lower Mancos into six members (table 1), from oldest to youngest, the Graneros, Bridge Creek Limestone, Blue Hill, Juana Lopez, Montezuma Valley, and Niobrara. The strata consist mainly of shale but can include chalk, calcarenite, siltstone, sandstone, and bentonite; commonly, they enclose a variety of concretions. In outcrops, the members are distinguished essentially by differences in their content of calcium carbonate (table 1) supplemented by the identity of constituent fossils.

Thicknesses of members from the measured outcrops vary in the region, which probably reflects lateral changes in facies and possibly the effects of truncation at disconformities in the stratigraphic sequence. Disconformities might mark the bases of the Juana Lopez and the Niobrara Members. Furthermore, the members at several places are poorly exposed, and dips used for the measurements may be incorrect. The Graneros Member, measured at five localities, ranges in thickness from $35 \mathrm{ft}$ at the Red Rock section to $85 \mathrm{ft}$ at the Peach Valley section. The Bridge Creek Limestone is $42 \mathrm{ft}$ thick near Mack, $50 \mathrm{ft}$ thick near Uncompahgre, and $140 \mathrm{ft}$ thick at the Red Rock section. All of the Blue Hill Member was described at five of the selected outcrops where it ranges in thickness from about $100 \mathrm{ft}$ near Olathe to $217 \mathrm{ft}$ near Uncompahgre. The Juana Lopez is as much as $120 \mathrm{ft}$ thick near Mack, but at five other outcrops to the southeast it is 40 to $86 \mathrm{ft}$ thick. The Montezuma Valley Member is as much as $115 \mathrm{ft}$ thick near Mack and is $52 \mathrm{ft}$ thick at Alkali Creek and $100 \mathrm{ft}$ thick near Olathe.

\section{Reference Cited}

Molenaar, C.M., Cobban, W.A., Merewether, E.A., Pillmore, C.L., Wolfe, D.G., and Holbrook, J.M., 2002, Regional stratigraphic cross sections of Cretaceous rocks from east-central Arizona to the Oklahoma Panhandle: U.S. Geological Survey Miscellaneous Field Studies Map 2382. 


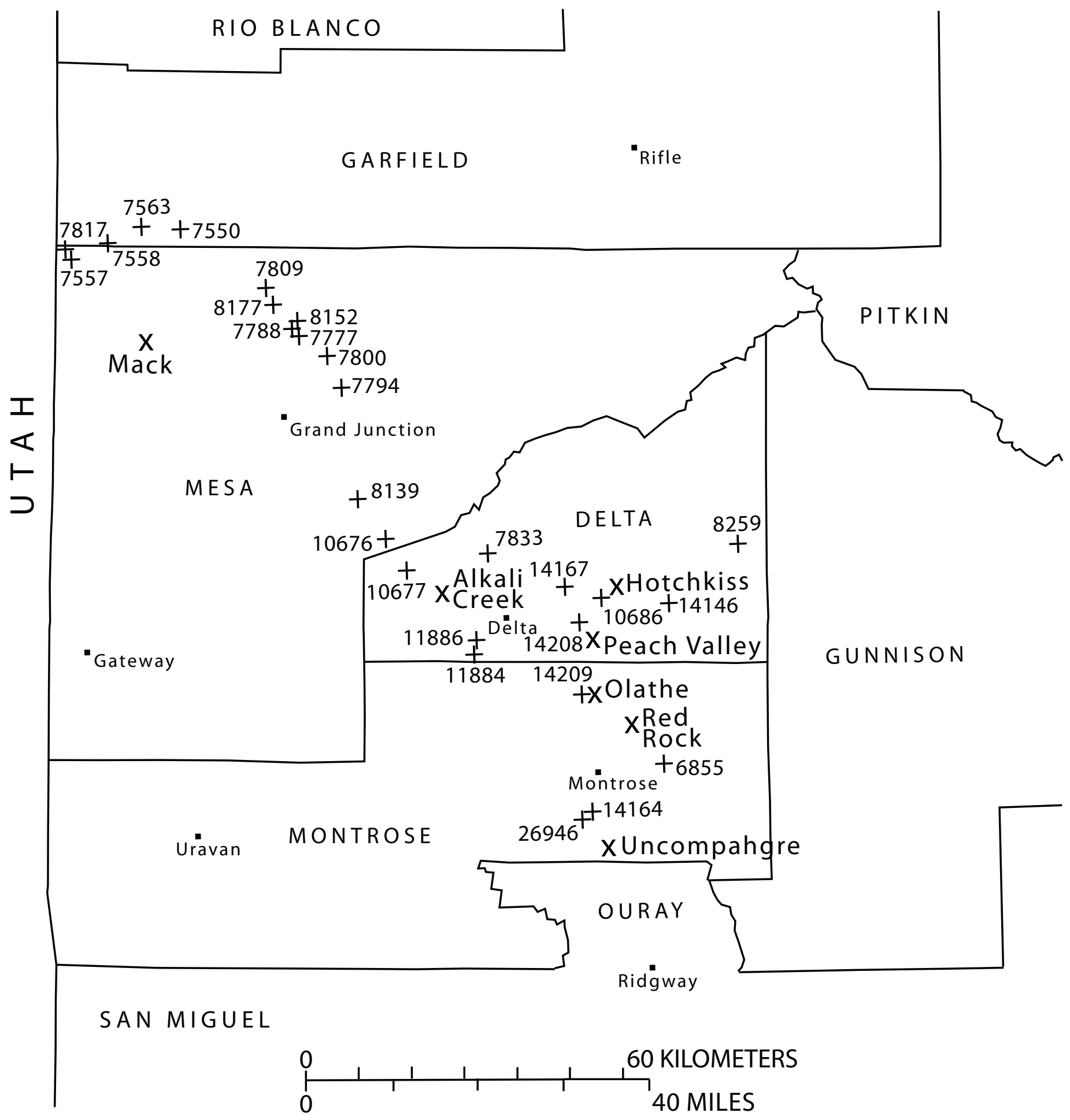

Figure 1. Measured outcrops $(X)$ and fossil localities (+) in Upper Cretaceous strata in counties of west-central Colorado. Numbers indicated specific fossil collections. 
Table 1. 3

Table 1. Members of Upper Cretaceous age in the lower part of the Mancos Shale of west-central Colorado. Names follow nomenclature of Molenaar and others (2002).

\begin{tabular}{|c|c|c|c|}
\hline MEMBER & DOMINANT LITHOLOGY & THICKNESSES (FT.) & AGE (STAGES) \\
\hline Niobrara & shale, calcareous & about 580 & late Turonian and Coniacian \\
\hline Montezuma Valley & shale, noncalcareous & 52 To 115 & late middle and early late Turonian \\
\hline Juana Lopez & shale and calcarenite & 40 To 120 & late middle Turonian \\
\hline Blue Hill & shale, noncalcareous & 101 TO 217 & early middle Turonian \\
\hline Bridge Creek Limestone & shale, calcareous & 42 T으 140 & late Cenomanian and early Turonian \\
\hline Graneros & shale, noncalcareous & 35 TO 101 & late Cenomanian \\
\hline
\end{tabular}


Table 2. Names and locations of selected outcrops of Upper Cretaceous strata and names of associated molluscan fossils in Delta, Mesa, and Montrose Counties, west-central Colorado. Names of members of the Mancos Shale follow nomenclature of Molenaar and others (2002).

\begin{tabular}{|c|c|c|c|c|c|c|c|}
\hline OUTCROP NAME & COUNTY & SEC. & TWN. & RNG. & USGS CATALOGUE NO. & MOLLUSCAN GUIDE FOSSILS & STRATIGRAPHIC UNIT \\
\hline \multirow[t]{3}{*}{ Alkali Creek } & Delta & SE, SW 25 & $4 \mathrm{~S}$. & $3 \mathrm{E}$. & $\begin{array}{l}\text { D10682 } \\
\text { D10683 } \\
\text { D10681 }\end{array}$ & $\begin{array}{l}\text { Prionocyclus quadratus } \\
\text { Inoceramus incertus } \\
\text { Prionocyclus quadratus } \\
\text { Inoceramus incertus } \\
\text { Scaphites whitfieldi } \\
\text { Inoceramus perplexus }\end{array}$ & $\begin{array}{l}\text { Niobrara Mbr.-Mancos Sh. } \\
\text { Niobrara Mbr.-Mancos Sh. } \\
\text { Montezuma Valley Mbr.-Mancos Sh. }\end{array}$ \\
\hline & & & & & D10679 & $\begin{array}{l}\text { Scaphites whitfieldi } \\
\text { Prionocyclus novimexicanus } \\
\text { Baculites yokoyamai } \\
\text { Inoceramus perplexus } \\
\text { Scaphites warreni } \\
\text { Prionocyclus wyomingensis } \\
\text { Inoceramus dimidius }\end{array}$ & $\begin{array}{l}\text { Montezuma Valley Mbr.-Mancos Sh. } \\
\text { Juana Lopez Mbr.-Mancos Sh. }\end{array}$ \\
\hline & -- & - & & & $\begin{array}{l}\mathrm{D} 1 \overline{06} \overline{8} \overline{5} \\
\mathrm{D} 10684\end{array}$ & $\begin{array}{l}\text { Prionocyclus macombi } \\
\text { Lopha lugubris } \\
\text { Inoceramus dimidius } \\
\text { Prionocyclus hyatti } \\
\text { Inoceramus howelli }\end{array}$ & $\begin{array}{l}\text { Juana Lopez Mbr.-Mancos S̄h. } \\
\text { Blue Hill Mbr.-Mancos Sh. }\end{array}$ \\
\hline Hotchkiss & Delta & $\begin{array}{l}\text { NE, NW } 31 \\
\text { SW, NE } 36\end{array}$ & $\begin{array}{l}14 S . \\
14 S .\end{array}$ & $\begin{array}{l}93 \mathrm{~W} . \\
94 \mathrm{~W} .\end{array}$ & $\begin{array}{l}\text { D14167 } \\
\text { D8257 } \\
\text { D14166 } \\
\text { D14165 } \\
\end{array}$ & $\begin{array}{l}\text { Inoceramus perplexus } \\
\text { Prionocyclus novimexicanus } \\
\text { Scaphites whitfieldi } \\
\text { Prionocyclus macombi } \\
\text { Inoceramus dimidius } \\
\text { Lopha lugubris } \\
\text { Inoceramus dimidius } \\
\text { Lopha lugubris } \\
\text { Pycnodonte aff. P. kellumi } \\
\end{array}$ & $\begin{array}{l}\text { Montezuma Valley Mbr.-Mancos Sh. } \\
\text { Juana Lopez Mbr.-Mancos Sh. } \\
\text { Bridge Creek Limestone Mbr.-Mancos Sh. }\end{array}$ \\
\hline Mack & Mesa & $\begin{array}{l}\text { SE, NW } 31 \\
\text { NW, NW } 5\end{array}$ & $2 \mathrm{~N}$. & $\begin{array}{l}3 W . \\
3 W .\end{array}$ & $\begin{array}{l}\text { D10442 } \\
\text { D10446 }\end{array}$ & $\begin{array}{l}\text { Scaphites whitfieldi } \\
\text { Inoceramus perplexus } \\
\text { Baculites yokoyamai } \\
\text { Prionocyclus macombi } \\
\text { Inoceramus dimidius } \\
\text { Lopha lugubris }\end{array}$ & $\begin{array}{l}\text { Montezuma Valley Mbr.-Mancos Sh. } \\
\text { Juana Lopez Mbr.-Mancos Sh. }\end{array}$ \\
\hline- & -1 & $\begin{array}{l}\overline{N W} \bar{N} \bar{N} \bar{W} 3 \overline{1} \\
N W, N W 31\end{array}$ & $2 \mathrm{~N}$. & $3 \mathrm{~W}$ & D10440 & $\begin{array}{l}\text { Collignoniceras woollgari } \\
\text { Pinna petrina } \\
\text { Laternula lineata } \\
\text { Pycnodonte newberryi }\end{array}$ & $\begin{array}{l}\text { Blue Hill Mbr.-Mancos Sh. } \\
\text { Bridge Creek Limestone Mbr.-Mancos Sh. }\end{array}$ \\
\hline Olathe & Montrose & SW, SE 9 & $50 \mathrm{~N}$. & $9 \mathrm{~W}$. & D14168 & Pycnodonte newberryi & Bridge Creek Limestone Mbr.-Mancos Sh. \\
\hline Peach Valley & Delta & $\begin{array}{l}\text { SE } 33 \\
\text { SW } 34\end{array}$ & $\begin{array}{l}15 S . \\
15 S .\end{array}$ & $\begin{array}{l}94 \mathrm{~W} . \\
94 \mathrm{~W} .\end{array}$ & $\begin{array}{l}\text { D11894 } \\
\text { D11893 } \\
\text { D11892 }\end{array}$ & $\begin{array}{l}\text { Inoceramus dimidius } \\
\text { Inoceramus dimidius } \\
\text { Lopha lugubris } \\
\text { Prionocyclus macombi } \\
\text { Inoceramus dimidius }\end{array}$ & $\begin{array}{l}\text { Juana Lopez Mbr.-Mancos Sh. } \\
\text { Juana Lopez Mbr.-Mancos Sh. } \\
\text { Blue Hill Mbr.-Mancos Sh. }\end{array}$ \\
\hline & & SE 34 & $15 S$. & $94 \mathrm{~W}$. & $\begin{array}{l}\text { D11891 } \\
\text { D11890 } \\
\text { D11889 } \\
\text { D11888 }\end{array}$ & $\begin{array}{l}\text { Prionocyclus hyatti } \\
\text { Inoceramus dimidius } \\
\text { Lopha lugubris } \\
\text { Pycnodonte newberryi } \\
\text { Johnsonites sulcatus } \\
\text { Borissiakoceras compressum } \\
\text { Inoceramus macconnelli }\end{array}$ & $\begin{array}{l}\text { Blue Hill Mbr.-Mancos Sh. } \\
\text { Blue Hill Mbr.-Mancos Sh. } \\
\text { Bridge Creek Limestone Mbr.-Mancos Sh. } \\
\text { Graneros Mbr.-Mancos Sh. }\end{array}$ \\
\hline Red Rock & Montrose & SE, SW 29 & $50 \mathrm{~N}$ & $8 W$. & $\begin{array}{l}\text { D14150 } \\
\text { D14149 }\end{array}$ & $\begin{array}{l}\text { Inoceramus dimidius } \\
\text { Lopha lugubris } \\
\text { Pseudoperna congesta } \\
\text { Inoceramus sp. }\end{array}$ & $\begin{array}{l}\text { Juana Lopez Mbr.-Mancos Sh. } \\
\text { Bridge Creek Limestone Mbr.-Mancos Sh. }\end{array}$ \\
\hline Uncompahgre & Montrose & NE, NE 3 & $47 \mathrm{~N}$ & $9 W$. & $\begin{array}{l}\text { D11883 } \\
\text { D11880 }\end{array}$ & $\begin{array}{l}\text { Prionocyclus macombi } \\
\text { Scaphites warreni } \\
\text { Inoceramus dimidius } \\
\text { Prionocyclus macombi } \\
\text { Inoceramus dimidius } \\
\text { Lopha lugubris }\end{array}$ & $\begin{array}{l}\text { Juana Lopez Mbr.-Mancos Sh. } \\
\text { Juana Lopez Mbr.-Mancos Sh. }\end{array}$ \\
\hline & & & & & $\begin{array}{l}\text { D11879 } \\
\text { D11878 } \\
\text { D11882 } \\
\text { D11881 }\end{array}$ & $\begin{array}{l}\text { Prionocyclus macombi } \\
\text { Scaphites carlilensis } \\
\text { Pycnodonte newberryi } \\
\text { Pycnodonte newberryi } \\
\text { Plicatula sp. }\end{array}$ & $\begin{array}{l}\text { Blue Hill Mr.-Mancos Sh. } \\
\text { Bridge Creek Limestone Mbr.-Mancos Sh. } \\
\text { Bridge Creek Limestone Mbr.-Mancos Sh. } \\
\text { Graneros Mbr.-Mancos Sh. }\end{array}$ \\
\hline
\end{tabular}


Table 3.

Table 3. Molluscan fossil localities in Upper Cretaceous strata in Delta, Garfield, Mesa, and Montrose Counties, westcentral Colorado. Names of members of the Mancos Shale follow nomenclature of Molenaar and others (2002).

\begin{tabular}{|c|c|c|c|c|c|c|}
\hline \begin{tabular}{|l} 
FOSSIL COLLECTIONS, \\
USGS CATALOGUE NO. \\
\end{tabular} & COUNTY & SEC. & TWN. & RNG. & \begin{tabular}{|l} 
MOLLUSCAN \\
GUIDE FOSSILS \\
\end{tabular} & $\begin{array}{l}\text { STRATIGRAPHIC UNITS } \\
\text { (Sh.=shale; } \text { Mbr.=member) }\end{array}$ \\
\hline \begin{tabular}{|l|}
26946 \\
D2040 \\
D6855 \\
D14164 \\
D14209 \\
\end{tabular} & Montrose & $\begin{array}{c}19 \\
30 \\
\text { NW, SW } 23 \\
\text { NW, NW } 20 \\
\text { NW 8 } \\
\end{array}$ & $\begin{array}{l}48 \mathrm{~N} . \\
48 \mathrm{~N} . \\
49 \mathrm{~N} . \\
48 \mathrm{~N} . \\
50 \mathrm{~N}\end{array}$ & $\begin{array}{l}9 W . \\
9 W . \\
8 W . \\
9 W . \\
9 W .\end{array}$ & $\begin{array}{l}\text { Plesiacanthoceras wyomingense } \\
\text { Conlinoceras tarrantense } \\
\text { Baculites obtusus } \\
\text { Pycnodonte aff. P. kellumi } \\
\text { Magadiceramus stantoni } \\
\end{array}$ & $\begin{array}{l}\text { Dakota Sandstone } \\
\text { Dakota Sandstone } \\
\text { Mancos Shale } \\
\text { Bridge Creek Limestone Mbr.-Mancos Sh. } \\
\text { Mancos Shale } \\
\end{array}$ \\
\hline $\begin{array}{l}\text { D7833 } \\
\text { D7835 } \\
\text { D7836 } \\
\text { D7837 }\end{array}$ & Delta & NE, SW 10 & $14 \mathrm{~S}$. & $96 \mathrm{~W}$. & $\begin{array}{l}\text { Baculites obtusus } \\
\text { Baculites asperiformis } \\
\text { Baculites asperiformis } \\
\text { Baculites asperiformis }\end{array}$ & Mancos Shale \\
\hline $\begin{array}{l}\text { D8142 } \\
\text { D8143 } \\
\text { D8256 } \\
\text { D8259 } \\
\text { D10677 }\end{array}$ & & $\begin{array}{l}\text { NE, SE } 10-7 \\
\text { SW, NE } 36 \\
\text { SE, NE } 4 \\
\text { SE, SW } 8\end{array}$ & $\begin{array}{l}\overline{1} \overline{4} \bar{S} \\
14 S . \\
14 S . \\
4 S .\end{array}$ & $\begin{array}{l}96 \bar{W} . \\
94 \mathrm{~W} . \\
91 \mathrm{~W} . \\
3 \mathrm{E} .\end{array}$ & $\begin{array}{l}\text { Baculites perplexus } \\
\text { Baculites perplexus } \\
\text { Pycnodonte } n \text {. sp. } \\
\text { Baculites perplexus } \\
\text { Prionocyclus macombi } \\
\text { Inoceramus dimidius } \\
\text { Lopha lugubris }\end{array}$ & $\begin{array}{l}\text { Mancos Shale } \\
\text { Mancos Shale } \\
\text { Juana Lopez Member-Mancos Shale }\end{array}$ \\
\hline $\begin{array}{l}\mathrm{D} \overline{1} \overline{06} \overline{7} \overline{8} \\
\text { D10686 } \\
\text { D11884 } \\
\text { D11885 }\end{array}$ & & $\begin{array}{l}\text { SE, NW } 2 \\
\text { SE, SE } 7 \\
\text { SW } 33\end{array}$ & $\begin{array}{l}15 S . \\
51 N . \\
15 S .\end{array}$ & $\begin{array}{l}94 \mathrm{~W} . \\
11 \mathrm{~W} \\
96 \mathrm{~W}\end{array}$ & $\begin{array}{l}\text { Prionocyclus hyatti } \\
\text { Pycnodonte aff. P. kellumi } \\
\text { Pycnodonte newberryi } \\
\text { Inoceramus howelli } \\
\text { Exogyra sp. }\end{array}$ & $\begin{array}{l}\text { Blue Hill Member-Mancos Şhale } \\
\text { Bridge Creek Limestone Mbr.-Mancos Sh. } \\
\text { Bridge Creek Limestone Mbr.-Mancos Sh. } \\
\text { Blue Hill Member-Mancos Shale }\end{array}$ \\
\hline \begin{tabular}{|l} 
Di $1 \overline{1} 8 \overline{8} \overline{6}$ \\
D14146 \\
D14167 \\
D14208 \\
\end{tabular} & & $\begin{array}{l}\text { SE, SW } 7 \\
\text { NE, NW } 31 \\
\text { SE, SE } 21\end{array}$ & $\begin{array}{l}15 \bar{S} . \\
15 \mathrm{~S} . \\
14 \mathrm{~N} . \\
15 \mathrm{~S} .\end{array}$ & $\begin{array}{l}96 \bar{W} . \\
92 \mathrm{~W} . \\
94 \mathrm{~W} . \\
94 \mathrm{~W}\end{array}$ & $\begin{array}{l}\text { Prionocyclus macombi } \\
\text { Inoceramus dakotensis } \\
\text { Scaphites whitfieldi } \\
\text { Prionocyclus novimexicanus } \\
\text { Inoceramus perplexus } \\
\text { Pycnodonte aff. P. kellumi }\end{array}$ & $\begin{array}{l}\text { Juana Lopez Member-Mancos Shale } \\
\text { Juana Lopez Member-Mancos Shale } \\
\text { Montezuma Valley Mbr.-Mancos Shale } \\
\text { Mancos Shale }\end{array}$ \\
\hline $\begin{array}{l}\text { D7557 } \\
\text { D7777 } \\
\text { D7783 } \\
\text { D7786 } \\
\text { D7788 } \\
\text { D7794 } \\
\text { D7795 }\end{array}$ & Mesa & $\begin{array}{l}\text { NE } 29 \\
\text { NW, SE } 2 \\
\text { SE, NE } 2 \\
\text { NW, NW } 34 \\
\text { NE } 25\end{array}$ & $\begin{array}{l}8 S . \\
10 S . \\
10 S . \\
9 S . \\
1 \mathrm{~N} .\end{array}$ & $\begin{array}{l}105 \mathrm{~W} . \\
100 \mathrm{~W} . \\
100 \mathrm{~W} . \\
100 \mathrm{~W} . \\
1 \mathrm{E} .\end{array}$ & $\begin{array}{l}\text { Haresiceras natronense } \\
\text { Baculites asperiformis } \\
\text { Baculites perplexus } \\
\text { Baculites gilberti } \\
\text { Baculites asperiformis } \\
\text { Baculites sp. (weak flank ribs) } \\
\text { Baculites obtusus }\end{array}$ & $\begin{array}{l}\text { Mancos Shale } \\
\text { Mancos Shale } \\
\text { Mancos Shale } \\
\text { Mancos Shale } \\
\text { Mancos Shale }\end{array}$ \\
\hline $\begin{array}{l}\text { D7800 } \\
\text { D7809 } \\
\text { D7812 } \\
\text { D7817 } \\
\text { D7820 } \\
\text { D7821 } \\
\text { D7823 }\end{array}$ & & $\begin{array}{l}\text { SW, } \bar{N} \bar{W} \overline{3} \\
\text { SW } 7 \\
\text { NE } 18\end{array}$ & $\begin{array}{l}1 \bar{N} . \\
9 S . \\
8 S .\end{array}$ & $\begin{array}{l}-\overline{\mathrm{W}} \cdot- \\
100 \mathrm{~W} . \\
104 \mathrm{~W} .\end{array}$ & $\begin{array}{l}\text { Baculites perplexus } \\
\text { Baculites sp. (weak flank ribs) } \\
\text { Baculites asperiformis } \\
\text { Baculites sp. (weak flank ribs) } \\
\text { Baculites obtusus } \\
\text { Baculites mclearni } \\
\text { Baculites asperiformis }\end{array}$ & $\begin{array}{l}\text { Mancos Shale } \\
\text { Mancos Shale } \\
\text { Mancos Shale }\end{array}$ \\
\hline $\begin{array}{l}\text { D8152 } \\
\text { D8155 } \\
\text { D8158 }\end{array}$ & & $\begin{array}{l}\text { SE, SE } 32 \\
\text { NW, SW } 26 \\
\text { SW, SW } 34\end{array}$ & $\begin{array}{l}\text { 9S. } \\
9 S .\end{array}$ & $\begin{array}{l}100 W . \\
100 W\end{array}$ & $\begin{array}{l}\text { Prionocyclus macombi } \\
\text { Lopha lugubris } \\
\text { Inoceramus dimidius } \\
\text { Baculites perplexus } \\
\text { Baculites obtusus } \\
\text { Baculites asperiformis }\end{array}$ & $\begin{array}{l}\text { Mancos Shale } \\
\text { Mancos Shale } \\
\text { Mancos Shale }\end{array}$ \\
\hline $\begin{array}{l}\mathrm{D} 8 \overline{16} \overline{-} \\
\mathrm{D} 8177 \\
\mathrm{D} 10676\end{array}$ & & $\begin{array}{l}\overline{N W}, \bar{N} E \overline{2} 9 \\
\text { NW, SE } 20 \\
\text { NW, NE } 26\end{array}$ & $\begin{array}{l}8 \mathrm{~S} . \\
9 \mathrm{~S} . \\
3 \mathrm{~S}\end{array}$ & $\begin{array}{l}2 \bar{W} . \\
100 \mathrm{~W} \\
2 \mathrm{E}\end{array}$ & $\begin{array}{l}\text { Baculites perplexus } \\
\text { Baculites asperiformis } \\
\text { Inoceramus howelli } \\
\text { Crassostrea sp. }\end{array}$ & $\begin{array}{l}\text { Mancos Shale } \\
\text { Mancos Shale } \\
\text { Blue Hill Member-Mancos Shale }\end{array}$ \\
\hline $\begin{array}{l}\text { D7550 } \\
\text { D7558 } \\
\text { D7560 } \\
\text { D7563 }\end{array}$ & Garfield & $\begin{array}{c}\text { SE } 4 \\
\text { NW, NE } 13 \\
\text { SE, NW } 3 \\
\end{array}$ & $\begin{array}{l}8 S . \\
8 S . \\
8 S .\end{array}$ & $\begin{array}{l}102 W . \\
105 W . \\
103 W .\end{array}$ & \begin{tabular}{|l} 
Baculites perplexus \\
Baculites obtusus \\
Baculites asperiformis \\
Baculites perplexus
\end{tabular} & $\begin{array}{l}\text { Mancos Shale } \\
\text { Mancos Shale } \\
\text { Mancos Shale }\end{array}$ \\
\hline
\end{tabular}




\section{OUTCROP DESCRIPTIONS}

In the following descriptions of outcropping strata, the stratigraphic units are in descending order (youngest to oldest).

\section{SECTION NEAR ALKALI CREEK, DELTA COUNTY}

Outcrops in SE1/4 SW1/4 sec. 25, T.4 S., R.3 E. (Point Creek 7 1/2-minute quadrangle). Measured and described by E.A. Merewether and W.A. Cobban in 1978.

Niobrara Member (part):

$$
\text { Thickness (feet) }
$$

11. Chalk, light brownish gray; fossiliferous at $2 \mathrm{ft}$ above base (D10682-Prionocyclus quadratus).

10. Shale, medium dark gray, calcareous; contains bentonite, $0.5 \mathrm{ft}$ thick, at $3 \mathrm{ft}$ above base. Fossiliferous at $5 \mathrm{ft}$ above base (D10683-Prionocyclus quadratus).

Thickness of Niobrara Member (part)

Montezuma Valley Member:

9. Shale, dark gray, calcareous; contains concretions. Fossiliferous concretions at base (D10681-Scaphites whitfieldi).

8. Shale, dark gray, slightly calcareous. Bentonite, $0.3 \mathrm{ft}$ thick, at top of unit. Fossiliferous at $27 \mathrm{ft}$ above base (D10680-Scaphites whitfieldi).

Thickness of Montezuma Valley Member

Juana Lopez Member:

7. Interlaminated shale, dark gray, and less siltstone, brownish gray, and calcarenite. Fossiliferous in uppermost $5 \mathrm{ft}$. (D10679-Scaphites warreni).

6. Interbedded shale, dark gray, and less siltstone and calcarenite.

Fossiliferous at $11 \mathrm{ft}$ above base

(D10685-Prionocyclus macombi).

Thickness of Juana Lopez Member 
SECTION NEAR ALKALI CREEK, DELTA COUNTY (continued)

Blue Hill Member (part):

5. Interlaminated shale, dark gray, and siltstone, greenish gray to light brown. Fossiliferous limonitic concretions $19 \mathrm{ft}$ above base (D10684-Prionocyclus hyatti).

4. Shale, dark gray; calcareous concretions at base and top.

3. Shale, dark gray, noncalcareous.

2. Siltstone, soft; poorly exposed; concretions at base and in upper half.

9

1. Shale, soft; poorly exposed.

$50+$

Thickness of Blue Hill Member (part) 
SECTION NEAR VILLAGE OF HOTCHKISS, DELTA COUNTY

Outcrops in NE1/4 NW1/4 sec. 31, T.14 S., R.93 W. (Lazear quadrangle). Measured and described by E.A. Merewether and D.A. Sawyer, September 22-26, 2003.

Niobrara Member (part): Thickness (feet)

5. Limestone.

Montezuma Valley Member:

4. Shale, dark gray, slightly calcareous. Fossiliferous beds at 15-20 ft below top (D14167-Scaphites whitfieldi).

Juana Lopez Member:

3. Interbedded shale, siltstone, and calcarenite. Fossiliferous in lower part (D8257-Prionocyclus macombi)

(D14166-Inoceramus dimidius).

Blue Hill Member:

2. Shale, gray; poorly exposed; includes bentonite and few concretions.

$60+$

Bridge Creek Limestone Member (part):

1. Shale, gray, calcareous; poorly exposed. Fossiliferous near top (D14165-Pycnodonte aff. P. kellumi). 
SECTION NEAR VILLAGE OF MACK, MESA COUNTY

Outcrops in NW1/4 NW1/4 sec. 31, T.2 N., R.3 W. (Mack 71/2-minute quadrangle). Measured and described by E.A. Merewether and W.A. Cobban on September 7, 1977.

Niobrara Member (part): Thickness (feet)

14. Shale, gray, calcareous.

Thickness of Niobrara Member (part)

Montezuma Valley Member:

13. Shale, medium to dark gray, slightly calcareous. Fossiliferous calcareous concretions in lowermost $50 \mathrm{ft}$ and uppermost $35 \mathrm{ft}$ (D10442-Scaphites whitfieldi).

Thickness of Montezuma Valley Member

Juana Lopez Member:

12. Interbedded shale, siltstone, and calcarenite; poorly exposed. Fossiliferous calcarenites in uppermost $30 \mathrm{ft}$

(D10446-Prionocyclus macombi).

11. Interbedded shale and siltstone; concretions in lowermost $10 \mathrm{ft}$.

Thickness of Juana Lopez Member

Blue Hill Member:

10. Shale and siltstone, poorly exposed. Concretions in siltstone at $12 \mathrm{ft}$ above base.

9. Siltstone, olive gray, slightly calcareous, soft; contains Ophiomorpha and other burrows.

8. Sandstone, very fine and fine grained, silty, partly calcareous; poorly exposed. Concretions at $11 \mathrm{ft}$ above base. Unit contains burrows.

7. Sandstone, medium gray to brownish gray, very fine grained, silty, calcareous; laminated with low-angle tabular cross-beds; contains horizontal and vertical smooth burrows. Fossiliferous (D10441- Collignoniceras woollgari).

6. Siltstone, brownish gray, soft, poorly exposed. 
SECTION NEAR VILLAGE OF MACK, MESA COUNTY (continued)

Bridge Creek Limestone Member:

5. Siltstone, brownish gray, calcareous, soft.

4. Shale, medium dark gray, calcareous, poorly exposed. Fossiliferous in basal $5 \mathrm{ft}$ (D10440-Pycnodonte newberryi).

3. Siltstone, clayey, calcareous; bentonite at top. Fossiliferous (Pycnodonte newberryi).

4

Thickness of Bridge Creek Limestone Member

Graneros Member:

2. Shale, dark gray, noncalcareous; silty shale, siltstone, and bentonite in uppermost $7 \mathrm{ft}$; poorly exposed.

Thickness of Graneros Member

Dakota Sandstone (part):

1. Sandstone; contains scattered black pebbles as much as 0.5 in long; beds about $1 \mathrm{ft}$ thick. Abundant, horizontal, smooth, branching burrows at top. 
SECTION NEAR OLATHE, MONTROSE COUNTY

Outcrops in SW1/4 SE1/4 sec. 9, T.50 N., R.9 W. (249209E, 4276795N), (Olathe 7 1/2-minute quadrangle). Measured and described by E.A. Merewether and D.A. Sawyer in September, 2003.

Niobrara Member (part):

Thickness (feet)

10. Shale, gray, calcareous; poorly exposed.

Montezuma Valley Member:

9. Shale, dark gray, noncalcareous; poorly exposed.

100

Thickness of Montezuma Valley Member

100

Juana Lopez Member:

8. Interbedded shale, siltstone, sandstone, and calcarenite; laminated and thin bedded.

7. Interbedded dark gray shale and light brownish gray siltstone;

laminated and thin bedded.

46

Thickness of Juana Lopez Member

86

Blue Hill Member:

6. Shale, dark gray, noncalcareous; concretions at base.

6

5. Shale, dark gray, noncalcareous. $\quad 50$

4. Shale, dark gray, noncalcareous; poorly exposed. 45

Thickness of Blue Hill Member

101

Bridge Creek Limestone Member:

3. Shale, gray, calcareous, poorly exposed; concretions at $50 \mathrm{ft}$ above base. Fossiliferous in uppermost $5 \mathrm{ft}$ (D14168-

Pycnodonte newberryi, from the upper Cenomanian zone of Euomphaloceras septemseriatum).

Thickness of Bridge Creek Limestone Member

Graneros Member:

2. Shale, dark gray, noncalcareous; concretions

at $15 \mathrm{ft}$ and $44 \mathrm{ft}$ above base; poorly exposed.

Thickness of Graneros Member

Dakota Sandstone (part):

1. Sandstone 


\section{Molluscan Fossils and Stratigraphic Descriptions from the Upper Cretaceous Mancos Shale, West-Central Colorado}

\section{SECTION IN PEACH VALLEY, DELTA COUNTY}

Outcrops in SE1/4 sec. 33 and SW1/4 sec. 34, T.15 S., R.94 W. (Olathe NW 71/2-minute quadrangle). Measured and described by E.A. Merewether and W.A. Cobban on June 23, 1982.

Juana Lopez Member:

Thickness (feet)

18. Interbedded shale, minor calcareous siltstone, and calcarenite; poorly exposed. Fossiliferous calcarenite in uppermost $5 \mathrm{ft}$ (D11894-Inoceramus dimidius).

17. Interbedded shale, medium gray, soft, and less siltstone, brownish gray, and calcarenite. Fossiliferous calcarenite at $6 \mathrm{ft}$ above base (D11893-Inoceramus dimidius).

Thickness of Juana Lopez Member

Blue Hill Member:

16. Interbedded shale, dark gray, and minor siltstone, light brownish gray, calcareous; fossiliferous (D11890-Prionocyclus macombi). Ferruginous concretions in uppermost $15 \mathrm{ft}$.

15. Interbedded shale, dark gray, noncalcareous, and minor siltstone, light brownish gray, calcareous. Calcareous septarian concretions in uppermost $3 \mathrm{ft}$.

14. Interbedded shale, dark gray, noncalcareous, well indurated, and minor siltstone, light brownish gray, calcareous, and calcarenite. Fossiliferous calcarenite near base of unit (D11890-Inoceramus dimidius). Fossiliferous at $4 \mathrm{ft}$. above base (D11891-Prionocyclus hyatti).

13. Shale, dark gray, noncalcareous; septarian concretions at top.

12. Shale, dark gray, noncalcareous; contains a few large cone-in-cone concretions.

11. Shale, gray, silty, noncalcareous, soft; cone-in-cone concretions at base.

10. Shale, gray, slightly calcareous; includes thin beds of siltstone in uppermost $5 \mathrm{ft}$; poorly exposed.

9. Shale, gray, poorly exposed. 
SECTION IN PEACH VALLEY, DELTA COUNTY (continued)

Bridge Creek Limestone Member:

8. Shale, gray, calcareous, soft; thin beds of bentonite in uppermost part. 8

7. Shale, gray, slightly calcareous, soft; poorly exposed. 14

6. Shale, gray, soft; bentonites at top and $2 \mathrm{ft}$ below top; poorly exposed.

Fossiliferous near top (D11889-Pycnodonte newberryi).

5. Concealed, probably shale, slightly calcareous; cone-in-cone concretions in lowermost part and calcareous concretions in uppermost part.

Thickness of Bridge Creek Limestone Member

Graneros Member:

4. Shale, gray, soft; poorly exposed.

3. Interbedded shale, dark gray, noncalcareous; minor siltstone, noncalcareous, with load casts and small burrows.

2. Interbedded siltstone and shale, soft; contain horizontal burrows.

Fossiliferous calcareous concretions at top

(D11888-Johnsonites sulcatus).

Thickness of Graneros Member

Dakota Sandstone (part):

1. Sandstone; upper surface displays abundant small and large ripple marks. 
SECTION NORTH OF PEACH VALLEY, DELTA COUNTY

Outcrops in S1/2 sec. 21, T.15 S., R.94 W. (Olathe NW 7 1/2-minute quadrangle). Measured and described by E.A. Merewether and D.A. Sawyer on May 14, 2004.

Niobrara Member (part):

Thickness (feet)

3. Shale, gray, calcareous; poorly exposed. Fossiliferous beds at about $55 \mathrm{ft}$ and $120 \mathrm{ft}$ above base.

Thickness of Niobrara Member (part)

$350+$

Montezuma Valley Member:

2. Shale, dark gray, noncalcareous; poorly exposed.

90

Thickness of Montezuma Valley Member

90

Juana Lopez Member (part):

1. Interbedded calcareous shale and siltstone, and calcarenite. 
SECTION NEAR RED ROCK CANYON, MONTROSE COUNTY

Outcrops in SE1/4 SW1/4 sec. 29, T.50 N., R.8 W. (Red Rock Canyon 71/2-minute quadrangle). Measured and described by E.A. Merewether and D.A. Sawyer on September 25, 2003.

Juana Lopez and Blue Hill Members, undivided:

Thickness (feet)

5. Shale, dark gray; calcarenite bed at top; poorly exposed.

Fossiliferous near top (D14150-Inoceramus dimidius).

Thickness of Juana Lopez and Blue Hill Members, undivided

135

Bridge Creek Limestone Member:

4. Shale, calcareous; poorly exposed. Pycnodontes in upper part.

3. Shale, dark gray, calcareous; calcareous concretions at top.

Thickness of Bridge Creek Limestone Member

Graneros Member:

2. Shale, brownish gray, noncalcareous.

Thickness of Graneros Member

Dakota Sandstone (part):

1. Sandstone, well indurated. 
SECTION NEAR UNCOMPAHGRE, MONTROSE COUNTY

Outcrops in NE1/4 NE1/4 sec. 3, T.47 N., R.9 W. (Colona 71/2-minute quadrangle). Measured and described by E.A. Merewether and W.A. Cobban in June, 1982.

Juana Lopez Member:

Thickness (feet)

16. Shale, dark olive gray, calcareous, soft, and minor calcarenite.

Fossiliferous in uppermost $15 \mathrm{ft}$ (D11883-Scaphites warreni).

15. Shale, olive gray, slightly calcareous, soft, and minor calcarenite; siltstone, $1.5 \mathrm{ft}$ thick, at top. Fossiliferous (D11880

-Prionocyclus macombi).

14. Shale, medium gray to olive gray, soft, noncalcareous in lowermost $5 \mathrm{ft}$, calcareous in uppermost $2 \mathrm{ft}$; concretions at top.

Thickness of Juana Lopez Member

Blue Hill Member:

13. Interlaminated shale, dark gray, noncalcareous, jarositic, and minor siltstone. Fossiliferous ferruginous concretions near top

(D11879-Scaphites carlilensis).

12. Shale, dark gray, silty, noncalcareous; few laminae and thin beds of siltstone in uppermost $5 \mathrm{ft}$; concretions $3 \mathrm{ft}$. below top.

11. Shale, dark gray, silty, noncalcareous; cone-in-cone concretions at base; septarian concretions at top.

10. Shale, dark gray, silty, noncalcareous; brownish gray, silty, calcareous concretions, $4 \mathrm{ft}$ in diameter, at base.

9. Concealed, probably shale.

8. Shale, dark gray, noncalcareous, soft; poorly exposed.

Thickness of Blue Hill Member

Bridge Creek Limestone Member:

7. Shale, gray, calcareous, and chalk, at top; bentonites in basal $4 \mathrm{ft}$. Fossiliferous at base (D11882-

Pycnodonte newberryi) and $5 \mathrm{ft}$ below top (D11878- Pycnodonte newberryi).

6. Concealed, probably shale, calcareous.

Thickness of Bridge Creek Limestone Member 
SECTION NEAR UNCOMPAHGRE, MONTROSE COUNTY (continued)

Graneros Member:

5. Concealed, probably shale; septarian concretions $3 \mathrm{ft}$ below top.

4. Concealed, probably shale; septarian concretions about $5 \mathrm{ft}$ below top.

3. Concealed, probably shale; septarian concretions at top.

2. Shale; poorly exposed; concretions near base and fossiliferous calcareous concretions at top (D11881-Plicatula sp.).

1. Bentonite, dusky orange.

2

Thickness of Graneros Member 